\title{
AN AGENT-BASED APPROACH FOR CONSTRUCTING SOFTWARE SYSTEMS OF VIRTUAL SIMULATION
}

\author{
Li Hongbing, Meng Bo, Chen Shifu \\ Nanjing University, P. R. China
}

\begin{abstract}
The design and construction of virtual reality environments involve technologies such as computer graphics, image processing, pattern recognition, intelligent interface, artificial intelligence, voice recognition, network, parallel processing, and high-performance computing. Some researchers insist that object-oriented and agent-oriented technologies are fundamental for virtual reality system design. This paper applies artificial intelligence to the design of virtual reality systems. Agents are constructed by using object-oriented methods and a set of underlying computing models, such as neural networks, genetic algorithms, expert systems, and plan managers. Some object-oriented frameworks of these computing models are presented to illustrate this approach. The example of a spaceship game will illustrate interactions among environments, agents, and underlying computing models. The approach and reusable class library presented herein can be applied to various virtual reality environment simulations and intelligent applications.
\end{abstract}

\section{Introduction}

The design and construction of virtual reality environments require technologies such as computer graphics, image processing, pattern recognition, intelligent interface, artificial intelligence, voice recognition, network, parallel processing, and high performance computing. Reference [1] suggests that object-oriented and agent-oriented technologies are the basic methodologies for designing virtual reality systems. In that book vision computing, neuron computing, and evolutionary computing are regarded as the primary advanced virtual computing models. Calderoni and Marcenar argue that many artificial life research areas resort to agent-based simulation, so they try to design a generic platform for allowing scientists to easily build simulation environments [2]. A similar effort has been made by Reignier et al, who also constructed a virtual reality multi-agent platform called AReVi [3].

This paper applies artificially intelligent technologies to the virtual reality worlds, and proposes an agent-based approach to virtual reality from the standpoint of software simulation system design. Agents are constructed by using an object-oriented method. At the same time we also present a set of underlying computing models such as neural networks, genetic algorithms, expert systems, and plan managers to support agents. A spaceship game illustrates the interactions among environment, agents, and underlying computing models.

In Section 2 we propose an abstract framework of virtual environments. All of the other work will be based on it. Section 3 presents those underlying computing models in detail. A spaceship game in Section 4 will demonstrate how to use the framework and computing models to develop a virtual environment simulation system. Section 5 concludes this paper. 


\section{Abstract Framework of Virtual Environments}

To explain how to construct agents and underlying computing models using the object-oriented method, we first propose an abstract framework of virtual environments as shown in Fig. 1. It includes agent modules and environment simulation modules (environment, geometric objects, and real objects are represented by the classes world, geometric_object and game_object respectively). These are the rules we abide by in designing those components: (1) The agent module and environment simulation modules are connected only loosely. (2) The agent module should be extensible and adaptable to any new environment. (3) The environment simulation modules are platform independent; they can be used on multiple platforms such as Windows, Unix X Window, OpenGL and Macintosh.

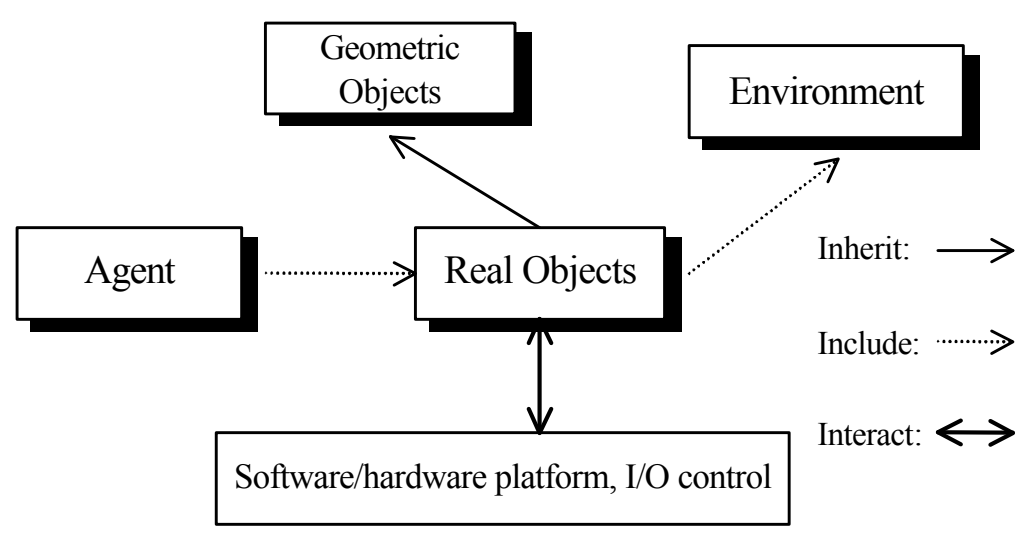

Fig.1. An Abstract Framework of virtual environments

Before giving the detailed class descriptions involved in those computing models, we first simply describe some classes called in them. The class geometric_object mainly deals with geometric models. The class game_object is a subclass of geometric_object. In addition to inheriting the data and methods from the base class, it also includes specific application data, the adding-agent method, the calling-agent method, and the changing self-status method.. The instance of the class game_object can refer to one or more instances of the class Agent.

The class World is a container class of the class game_object. An instance of it can contain one or more instances of the class game_object. The class Agent is a virtual base class. The main functions of it are to provide the interface for inherited classes and executing the agent's function run_agent, which supports the dynamic and real time behaviors of the simulation environment systems.

\section{Computing Models}

\subsection{NEURAL NETWORKS}

Neural networks perform well in the field of pattern recognition, especially in eliminating noise data and recognizing incomplete patterns [4][5]. In virtual environments these patterns are 
sensed by data and triggered by control data. The trained neural networks can be used to match the input and output data patterns.

In section 4 we use a trained neural network to compute the spaceship's speed. It uses the relative position to nearby objects as its input value. The description of the class Neural is shown in Fig. 2; it represents one simple neural network.

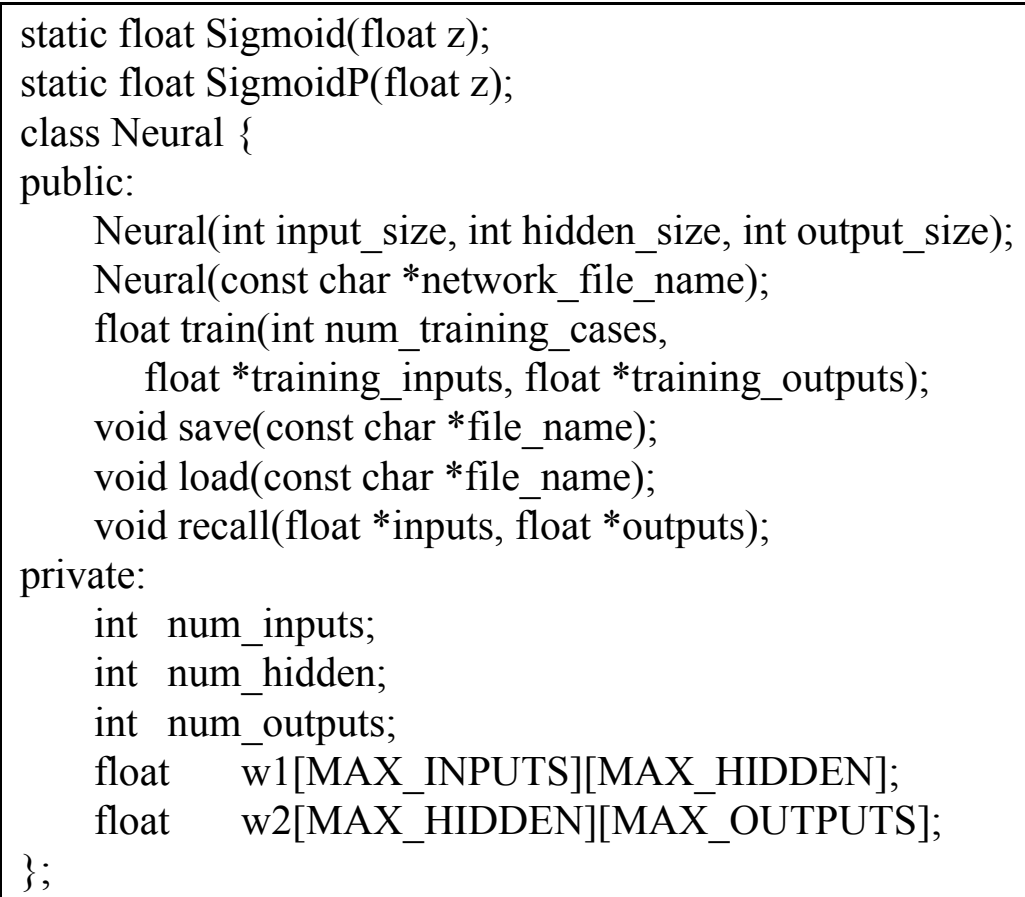

Fig. 2. Class Neural

The function Sigmoid limits the input values of all neural nodes to the specified bound, for example $(-0.5,0.5)$. It and SigmoidP are used to adjust the connection weights of networks during the training procedure. The first constructor function sets the number of neural nodes in input, output, and hidden layers, and it uses random numbers to set the connection weights of networks. The second constructor function constructs the network by using one file that contains the connection weights. The Train function is used to train the network and constantly change the weights until the sum of errors for all training examples is minimized. The Save and load functions are used to save and load the weights of trained networks. The Recall function can produce a set of outputs according to a group of neural nodes' inputs.

\subsection{GENETIC ALGORITHM}

A genetic algorithm is a search algorithm based on natural selection and natural inheritance. It applies a fitness function to each chromosome of the population. The chromosomes that have lower fitness are replaced by the new chromosomes that are generated by crossover and mutation functions among fit chromosomes. Each chromosome includes a specific number of genes. Often real applications represent a gene by only one bit. The two most frequently used operators of genetic algorithm are (1) the crossover operator, which generates new chromosomes through exchanging some genes of two fit chromosomes to replace the unfit chromosomes, and (2) the mutation operator, which changes some genes of those unfit chromosomes. 


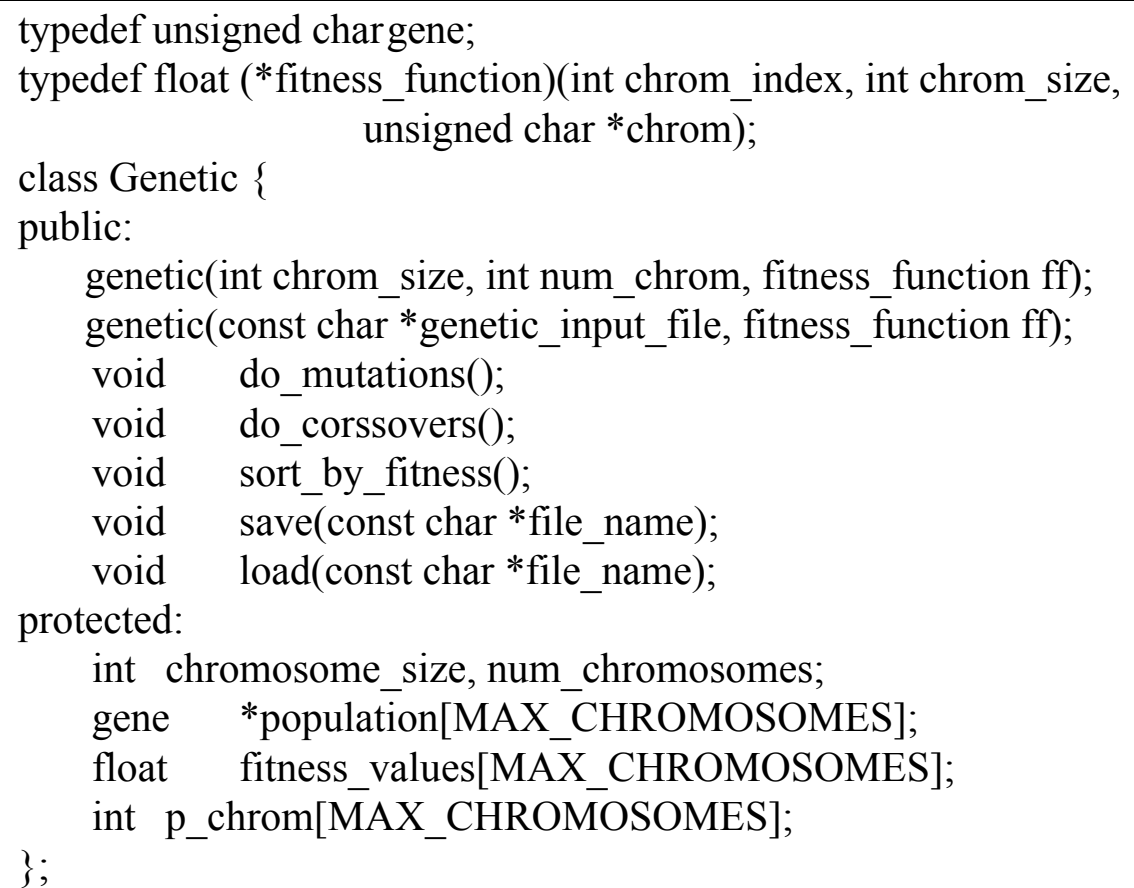

Fig. 3. Class Genetic

Section 4 will introduce a method of using chromosomes to save the spaceship's control strategies in a virtual environment as well as a method of using a genetic algorithm to control the spaceship. The description of the class Genetic is shown as Fig. 3. It represents one simple genetic algorithm. The function do_crossovers and the function do_mutations create new chromosomes in the population. The constructor function of the class Genetic uses random numbers as gene values to initialize the chromosomes. The function sort_by_fitness calls each chromosome's fitness function, sorts all the chromosomes according to the return values, and saves them in $p_{-}$chrom. The functions save and load are used to save and load the code of each chromosome in the population.

The instance of class Genetic must include a specific number of chromosomes and their related fitness functions. When using the class Genetic in Fig. 3, the method of encoding the chromosomes and providing a fitness function for each of them should be determined in advance.

\subsection{EXPERT SYSTEMS}

Expert systems separate domain knowledge from control. We implement them using a set of "if-then" rules. The separated inference engine interprets and executes those executable rules. It determines the executable rules and uses data of the current status. If the precondition part of a rule can be satisfied, then this rule will be regarded as executable, and the subsequent part of it can be executed by the inference engine. In order to realize a more applicable expert system, multiple knowledge representation methods, monotonic reasoning, fuzzy knowledge handling, and parsing analysis of semantic networks should also be supported [5][6]. 
In virtual environments an expert system is usually customized to a specific object. However, all instances of this group of objects can share the same instance of an expert system because an expert system is oriented to one group of objects rather than one specific object. Unlike the classes Neural and Genetic, the class ExpertSystem needs the knowledge of the classes World and game_object, but it will not be changed when the classes World and game_object have some changes.

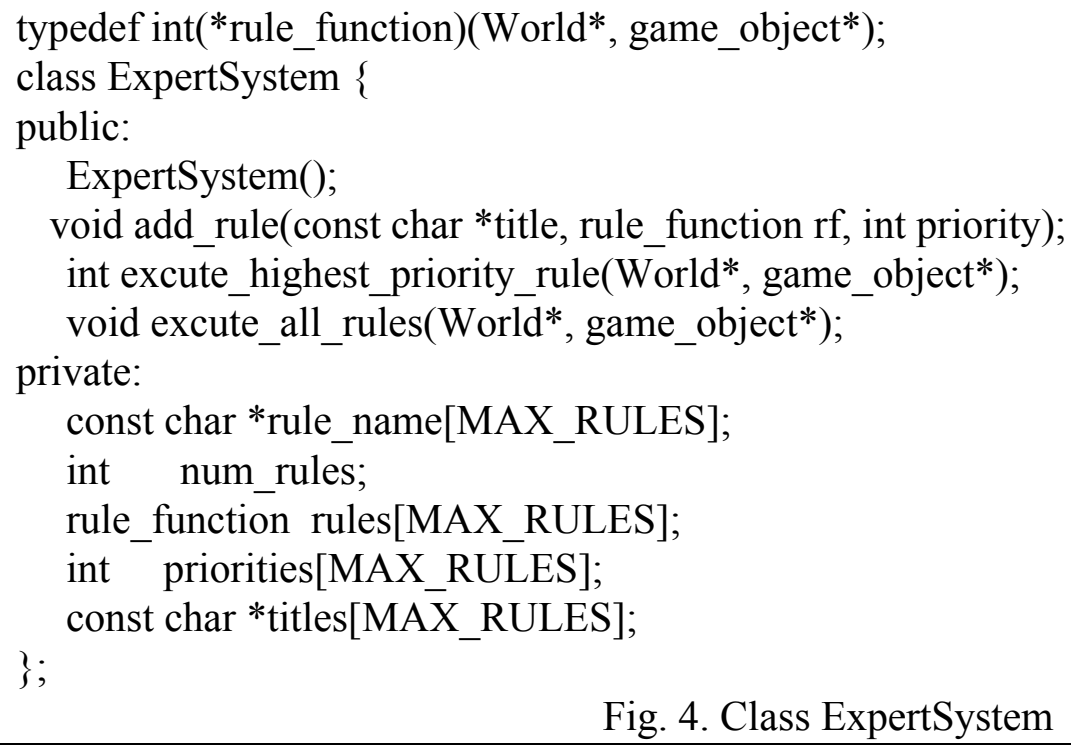

Class ExpertSystem is described in Fig. 4. The function add_rules will add rules to the instances of class, and the rules must match the function prototype

$$
\text { typedef int (*rule_function) (World*, game_object*); }
$$

A rule should provide at most two functions: (1) The first determines whether the precondition part of the rule matches current status; if it does not, it returns to FALSE immediately. (2) If the rule is executable, the subsequent part can be used to change the status of the instances that are presented by the classes World and game_object.

\subsection{PLAN MANAGER}

Plan managers are designed to handle plans that are integrated by three functions: (1) the condition judgment function that determines whether the precondition part of the plan can be matched with the current status; (2) the plan execution function that executes at each time step while the plan is active. This terminates when the termination judgment function returns to a TRUE value; (3) the termination judgment function that determines if the plan should be terminated.

Although the appearance of the class PlanManager is very similar to the class ExpertSystem, some differences still exist between them. Expert systems don't save their behaviors to the memo. These behaviors refer to the procedures executed between the previous time and the present time of the calling function to execute the highest priority rule or excute all rules. In contrast, plan managers do save the behaviors. Plan managers manage all status changes during the executing cycle. If specific actions are expected to be executed immediately by an agent, then the class ExpertSystem should be selected. If a sequence of actions is expected, then the class PlanManager is the appropriate choice. 


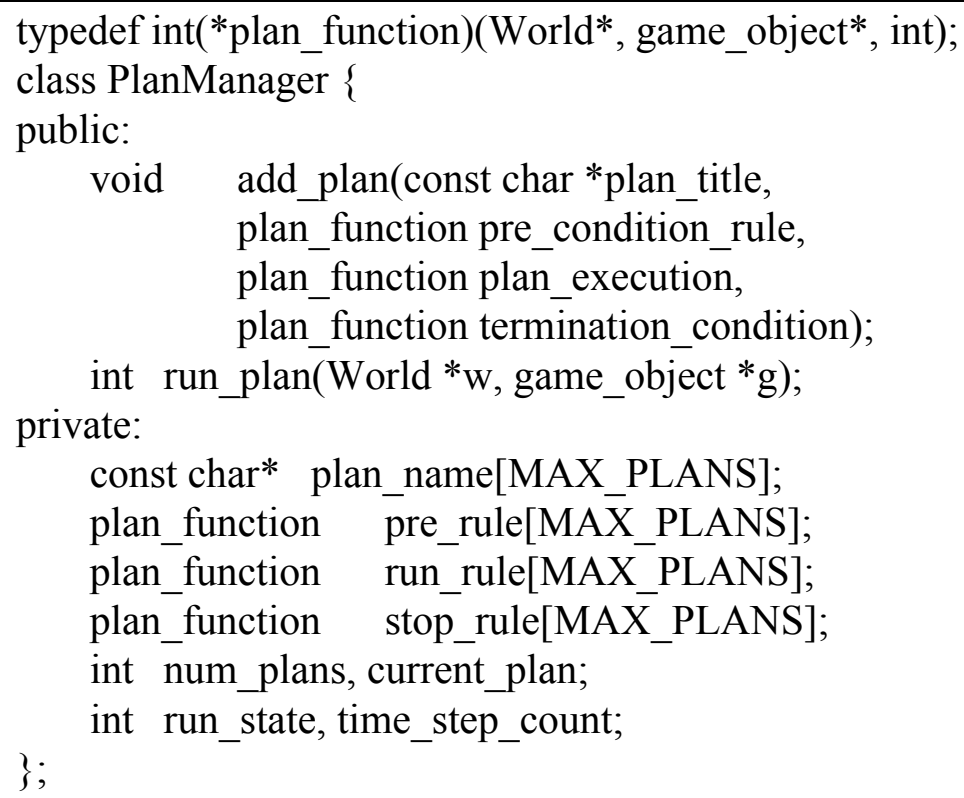

Fig. 5. Class PlanManager

The class PlanManager is described in Fig. 5. The function add_plan adds new plans to the instance of the class PlanManager. The function run_plan first judges if any plans are currently being executed. If not, each plan's condition judgment function is examined to judge if there is a proper plan to be executed.

\section{Spaceship Simulation}

\subsection{GAME DESCRIPTION}

There are three types of spaceships in the game: PlayerShip, ProcessorShip, and Corsair. PlayerShip has mines on asteroids, and a number of ProcessorShips can provide energy to PlayerShip in exchange for ore. The exchange process is proposed by PlayerShip, and ProcessorShip decides whether or not to exchange according to the amount of ore. If ProcessorShip has enough ore, it will not exchange with the PlayerShip. During the game, there are many Corsairs, and they keep stealing energy from the nearby PlayerShip and ProcessorShip. This game terminates when the PlayerShip's energy is depleted.

\subsection{NEURAL NETWORK NAVIGATION}

A neural network is used to simplify the problem by controlling the spaceship's speed and acceleration in specific directions. It uses different input values to describe the directions of $\mathrm{X}, \mathrm{Y}$, $Z$, and $-X,-Y$ and $-Z$. As the input and output values are restricted to $(-0.5,0.5)$, the positions of all other nearby spaceships should be expressed as input forms of the network. Then the real value outputs should be restored to the speeds and accelerations of the spaceships. The class NavigationAgent is used to navigate the PlayerShip and various ProcessorShips. It is a derived class from the class Agent and can call neural networks, expert systems, and plan managers. The class NavigationAgent is shown in Fig. 6. 


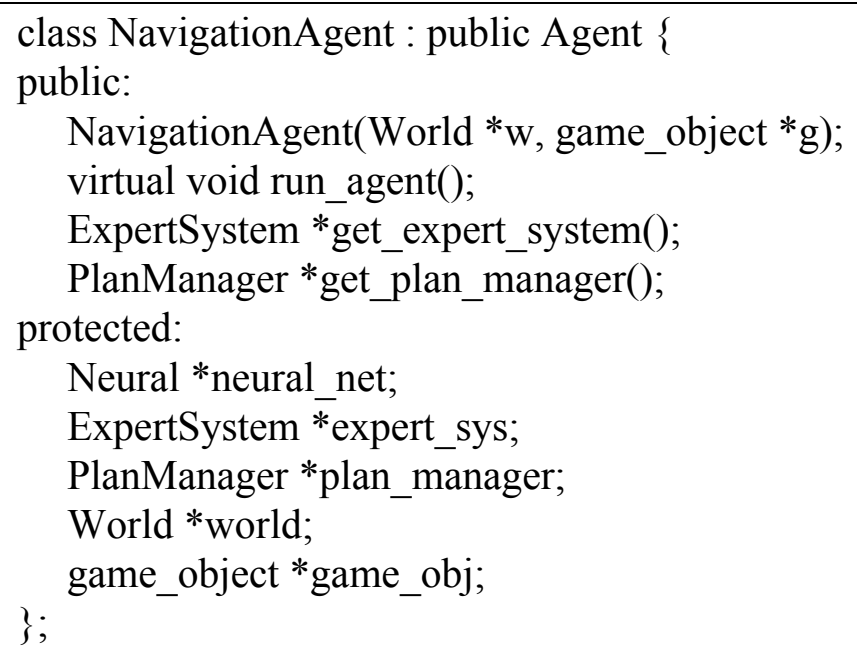

Fig. 6. Class NavigationAgent

The function run_agent first computes the distances between the spaceship and the nearest four objects (including other spaceships and asteroids) and converts the values to the input forms for the network. It then changes the speeds and accelerations according to the suggestion of the network. After that it calls the plan manager to judge if any executing plans are required. If there is no new plan at that point, the function execute_highest_priority_rule of the expert system will be called.

4.3 EXPERT SYSTEM

CONTROLLING PROCESSORSHIP

The class ProcessorShip represents the data and actions of ProcessorShip. It is a subclass of the class game_object and is shown as Fig. 7. The construction function of the class game_object specifies the shape and size for the construction of objects. After that, an instance of the class NavigationAgent is created to navigate ProcessorShip. At the same time, three rules are added to the expert system that are referred by the newly created navigation agent:

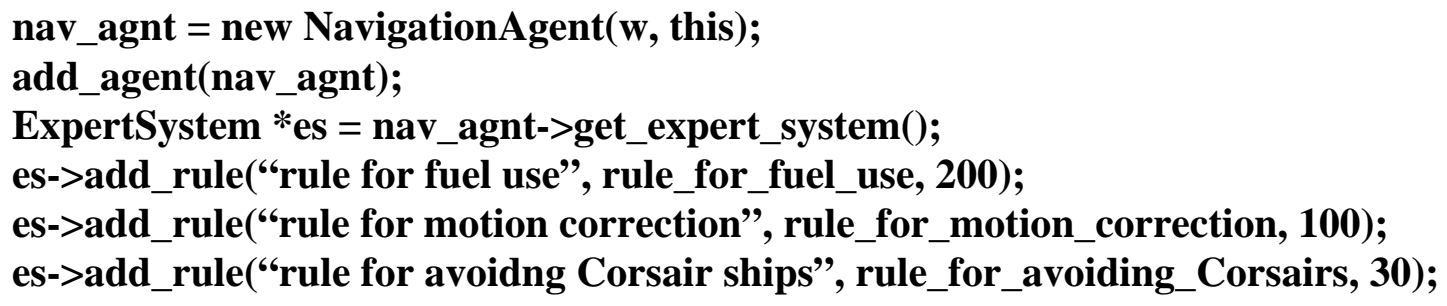

The function rule_for_fuel_use reduces the energy value at each time step. The function rule_for_motion_correction judges the environment status. If ProcessorShip is far away from the asteroid center, it will adjust its speed to approach the center. The function rule_for_avoiding_Corsairs will move ProcessorShip in the opposite direction when Corsair is near. 


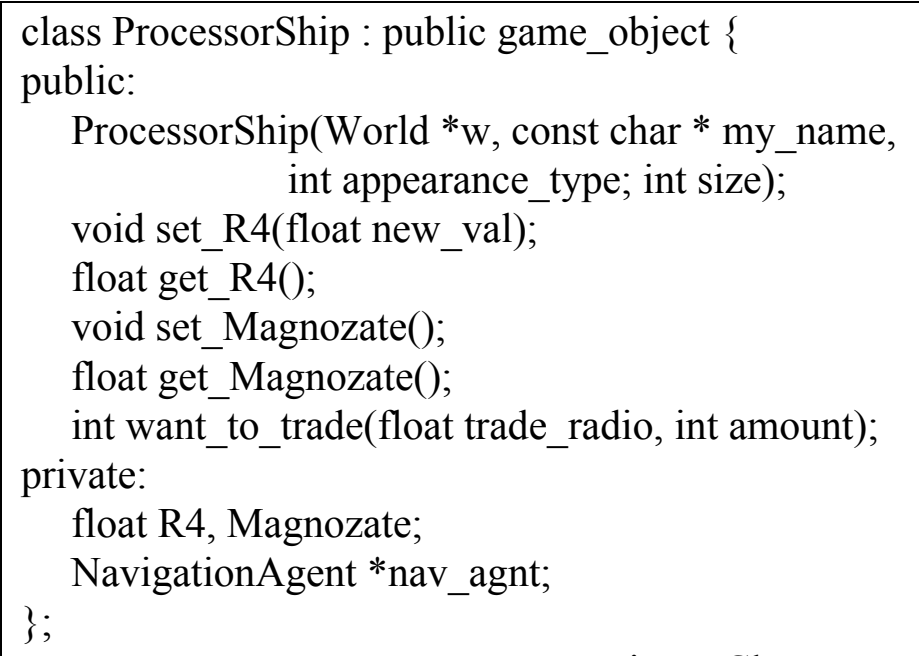

Fig. 7. Class ProcessorShip

\subsection{EXPERT SYSTEM AND PLAN \\ MANAGER AID PLAYERSHIP}

Like the class ProcessorShip that controls processor ships, the player ship can be aided by class PlayerShip, which is described in Fig. 8. It is also a subclass of the class game_object. The construction function specifies the shape and size of objects to be constructed. Then an instance where the class NavigationAgent will be created to aid PlayerShip. At the same time, five rules will be added to the expert system, referred by the newly created navigation agent:

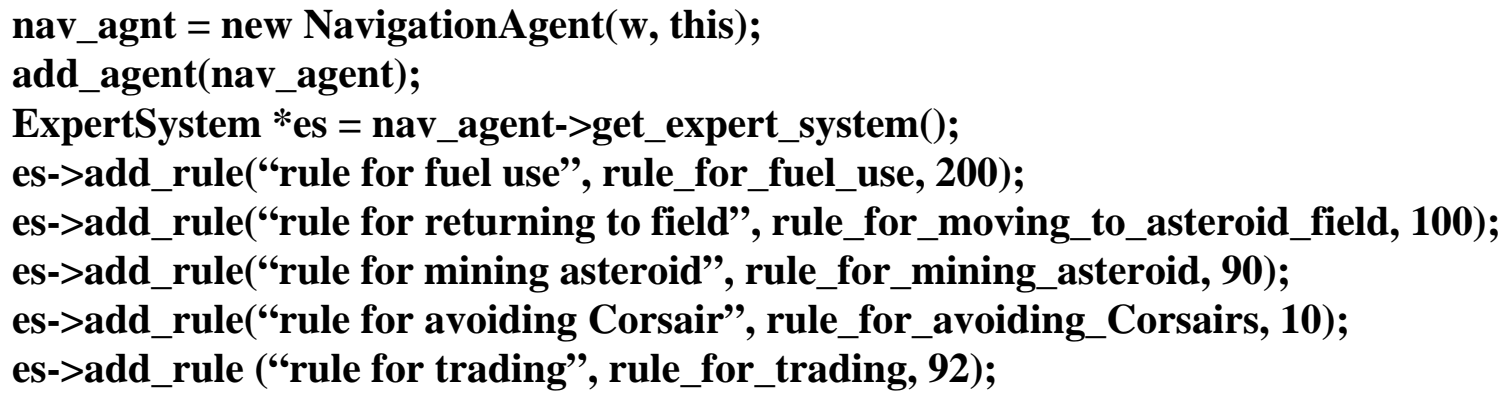

The function rule_for_fuel_use reduces PlayerShip's energy at each time step. If PlayerShip is far away from the asteroid center, the function rule_for_moving_to_asteroid_field will adjust its speed to approach the center. The function rule_for_mining_asteroid searches for the nearest asteroid to mine ore. The function rule_for_avoiding_Corsairs finds the nearby Corsairs and sets PlayerShip's speed to take it away from the Corsairs. The function rule_for_trading searches for the nearest processor ship, trying to exchange ore for energy. 


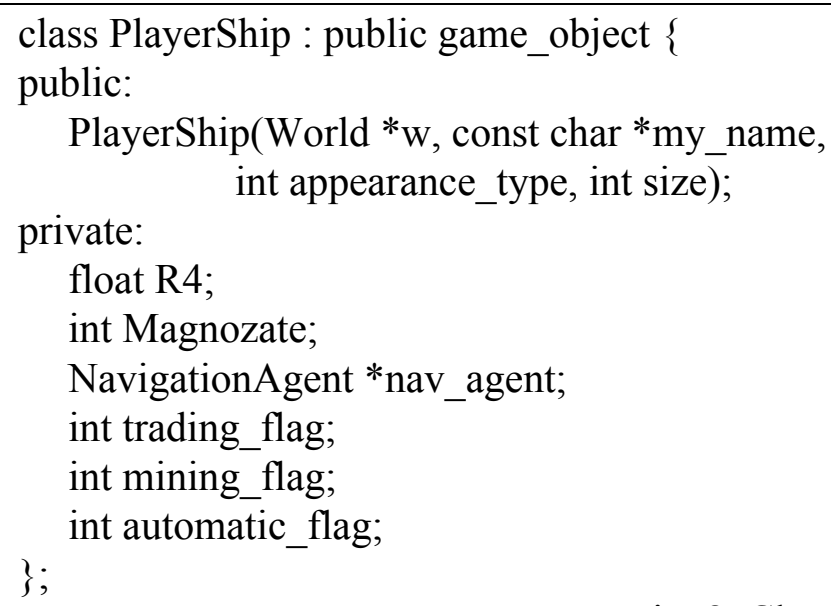

Fig. 8. Class PlayerShip

Except for the rules, the construction function of PlayerShip adds two plans to the instance of PlanManager.

\section{PlanManager *pm = nav_agnt->get_plan_manager () ; pm->add_plan(“"get ore", test_low_ore, go_to_asteroid, end_go_to_asteroid); pm->add_plan(“'trade ore”, test_trade_ore, go_to_pr_ship, end_go_to_pr_ship);}

These are the three plan functions for mining ore: (1) Test_low_ore creates a plan to move PlayerShip to mine ore from an asteroid when judging that PlayerShip's ore is low. (2) Go_to_asteroid moves PlayerShip to the asteroid. (3) End_go_to_asteroid terminates the plan if PlayerShip has approached the asteroid or the executing time of the plan is over the threshold.

\subsection{CHANGING CORSAIR STRATEGY DYNAMICALLY BY GA}

Corsair adopts a strategy different from PlayerShip and ProcessorShip. Each strategy is decided by three kinds of status parameters: (1) One where Corsair moves straight ahead or zigzags. (2) One where Corsair converts energy slowly from asteroids or steals energy quickly from PlayerShip or ProcessorShip. (3) One where Corsair moves slowly or quickly (and thus rapidly consumes energy).

Using 0 and 1 for the determination of the above three status parameters, we have eight possible strategies for each Corsair. Creating a chromosome of three genes for each Corsair satisfies the demand of the problem. The class Corsair is a subclass of the class Genetic. It is shown as Fig. 9. Only one instance of the class Corsair in our example needs to be created. This instance will include many chromosomes, and each chromosome represents the control strategy of one Corsair. The list in game_object has specified the appearance and status of each Corsair. 


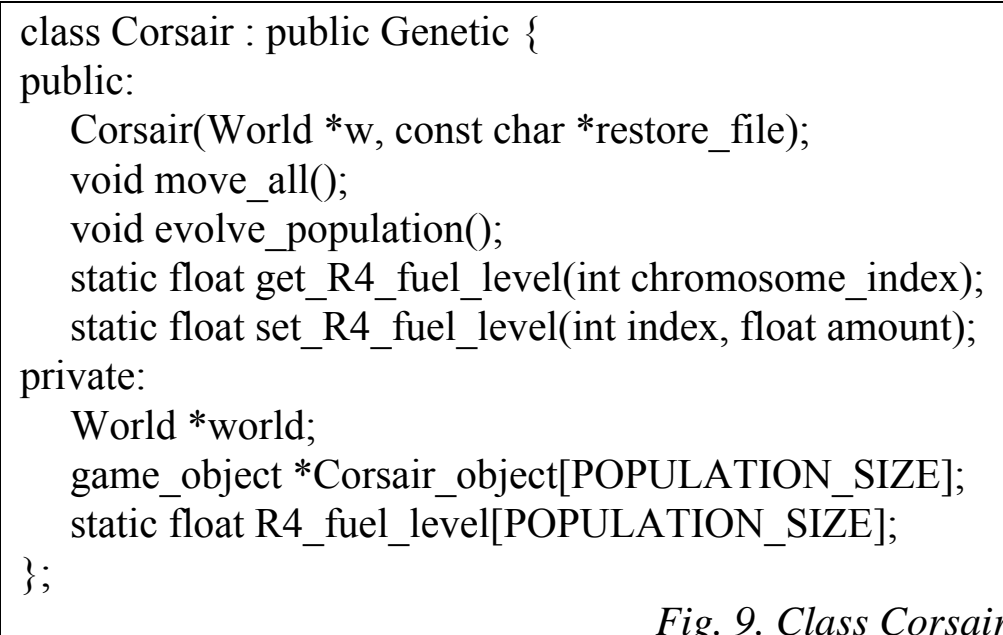

The function move_all changes all Corsairs' positions. The function evolve_population makes the population evolve periodically through the following three steps: (1) Sort all the chromosomes according to the fitness functions. (2) Apply the mutation operation to the unfit chromosomes. (3) Apply the crossover operation. For these Corsairs, we can simply take their energy values as fitness functions. If the energy of one Corsair is inadequate, its strategy is considered unfit.

\section{Conclusion}

The paper focuses on the realization of an agent and its underlying computing models based on object-oriented methodology. The ideas presented here can be applied to various virtual reality environment simulations and ordinary intelligent applications. Furthermore, the reusable and extensible classes proposed in this article such as the environment, the geometric objects, the real objects, the agent, the neural network, the expert system, the plan manager, and the genetic algorithm can be applied directly to related applications. However, those computing models are only simple prototypes because the main purpose of this paper is not to develop specific computing models. In practice, based on the demand of specific domain problems, new classes can be derived from those classes or directly extended to improve their functionality. In addition, this article mainly depends on the agents' ability to solve problems, and more complex problems can be solved if cooperation, coordination, and negotiation mechanisms among agents are introduced [10]. 


\section{REFERENCES}

[1] Wang Chengwei, Gao Wen, and Wang Xingren. Theory, Implementation and Application of Virtual Reality Technology, Tsinghua Press, Beijing, 1996.

[2] Calderoni, S., and Marcenac, P. "MUTANT: a multiagent toolkit for artificial life simulation". In: Proceedings of Technology of Object-Oriented Languages, TOOLS 26, 1998, pp. $218-229$.

[3] Reignier, P., Harrouet, F., Morvan, S., Tisseau, J., and Duval, T. "AReVi: A Virtual Reality Multiagent Platform", J.-C. Heudin (Ed.): Virtual Worlds 98, LNAI 1434, 1998, pp. 229-240.

[4] Chen Zhaoqian, Li Hongbing, Zhou Rong, and Chen Shifu. "Analysis and Improvement of FTART Algorithm”, Journal of Software, Vol.8, No.4, 1997, pp. 259-265.

[5] Masters T. Advanced Algorithms for Neural Networks, New York: John Wiley \& Sons, Inc., 1995.

[6] Koza, J. R. Genetic Programming, Cambridge, MA: MIT Press, 1992.

[7] Li Hongbing, Chen Zhaoqian, and Chen Shifu. "An Object-Oriented Expert System Development Tool”, Journal of Software, Vol. 8, Supplement, 1997, pp. 305-311.

[8] Medsker L R. Hybrid Neural Network and Expert Systems, Boston: Kluwer Academic Publishers, 1994.

[9] Ghallab M, Milani A. New Directions in AI Planning, Netherlands: IOS Press, 1996.

[10] Greg M P, O'Hare Nick R Jennings. Foundations of Distributed Artificial Intelligence, New York: John Wiley \& Sons, Inc., 1996. 


\section{BIOGRAPHIES}

Li Hongbing is on the academic staff of the State Key Laboratory for Novel Software Technology, Nanjing University. He received B.Sc., M.S., and Ph.D. degrees from Nanjing University, all in Computer Science. His current research interests include fuzzy systems, machine learning, neural networks, genetic algorithms, expert systems, multi-agent systems, and intelligent CAD. He has published more than 20 papers. He is now visiting the Department of Computing at the Hong Kong Polytechnic University as a research assistant.

\section{Contact information:}

Li Hongbing

Department of Computing

The Hong Kong Polytechnic University

Kowloon, Hong Kong

Phone: 852-27667312

Fax: $\quad 852-27740842$

Email: mailto:cshbli@,comp.polyu.edu.hk

Meng Bo is a Ph.D. student of the Computer Science and Technology Department, Nanjing University. He received an M.S. degree from Nanjing University in computer science. His current research interests include intelligent CAD and distributed artificial intelligence.

\section{Contact Information:}

Meng Bo

Computer Science and Technology Department

Nanjing University

Nanjing, P. R. China, 210093

Phone: 86-25-3593163

Fax: $\quad 86-25-3300710$

Email: mailto:russell@,263.net

Chen Shifu is a professor of the Computer Science and Technology Department, Nanjing University, once served as the Department Head. Now he is the Deputy Chairman of China Machine Learning Association and a senior fellow of the China Artificial Intelligence Association. His research interests include Machine Learning, Neural Network, Knowledge Engineering, Intelligent Agent, and Intelligent CAD.

\section{Contact Information:}

Chen Shifu

Computer Science and Technology Department

Nanjing University

Nanjing, P. R. China, 210093

Phone: 86-25-3593163

Fax: $\quad 86-25-3300710$

Email: mailto:chensf@,nju.edu.cn 